\title{
Ultrastructural pathology of human peritumoural oedematous cerebellar cortex
}

\author{
Orlando José Castejón \\ Biological Research Institute, Faculty of Medicine, Zulia University, Maracaibo, Venezuela
}

Folia Neuropathol 2016; 54 (2): 127-136

DOI: $10.5114 / f n .2016 .60057$

\begin{abstract}
Cerebellar cortical biopsies of the peritumoural region of seven patients with cerebellar haemangioma, mesencephalic meningioma, cerebellopontine astrocytoma, cerebellopontine meningioma, and medulloblastoma of cerebellar vermis were examined by means of conventional transmission electron microscopy. Granule cells showed oedematous cytoplasm and mitochondria. Swollen Golgi cells exhibited lipofuscin granules and intranuclear inclusions. Both neuron cell types displayed swollen dendritic digits synapsing with afferent mossy fibre endings. Degenerated myelinated axons corresponding to afferent mossy and climbing fibres and efferent Purkinje cell axons were observed at the granular layer. Dense and clear ischaemic Purkinje cells established degenerated synapses with swollen parallel fibre synaptic varicosities. Degenerated Purkinje cell recurrent axonal collaterals were found at the molecular layer. Swollen and clear Bergmann glial cell cytoplasm was observed closely applied to the oedematous clear and dark Purkinje cell body, dendritic trunk, secondary and tertiary dendritic branches. Swollen climbing fibre endings featured by numerous microtubules and neurofilaments, and a decreased number of synaptic vesicles were observed making degenerated axo-spinodendritic synapses with clear and swollen dendritic spines from Purkinje, Golgi, basket and stellate cell dendrites. Swollen stellate neurons showed oedematous mitochondria. Lipofuscin-rich astrocytes and reactive phagocytic astrocytes were observed. The latter appeared engulfing haematogenous proteinaceous oedema fluid. All cerebellar neurons showed stress endoplasmic reticulum dysfunction featured by focal dilated cisterns and detachment of associated ribosomes. Myelin sheath degeneration was related with oligodendrocyte degenerating hydropic changes. The peritumoural ischaemic cerebellar nerve and glial cell abnormalities were related with neurobehavioral changes, tremor, nystagmus, dismetry and gait disturbance observed in the patients examined. The ultrastructural pathological changes were correlated with the biochemical cascade induced by vasogenic and cytotoxic oedema, altered calcium homeostasis, increased glutamate excitotoxicity, oxidative stress and DNA damage.
\end{abstract}

Key words: human cerebellum, cerebellar neurons, cerebellar oedema, cerebellar tumours, electron microscopy.

\section{Introduction}

Some earlier research investigations have been reported on ultrastructural biology and pathology of cerebellar tumours $[14,15,16,27]$. Axonal torpedoes on Purkinje cells of the cerebellum have been observed at electron microscopic level in infantile neuroaxonal dystrophy, in two cases of brain tumours, and in a case of a 5 -year-old boy suffering from juvenile astrocytoma [21,24,32]. The presence of intranuclear filamentous inclusions in cerebellar 
Golgi cells was reported by us in three patients with cerebellar tumours [4].

However, very few electron microscopic studies have been devoted to study the cortical biopsies of the cerebellar peritumoral region taken during neurosurgical treatment of human cerebellar, cerebellopontine and mesencephalic tumours for systematically examining the alterations of cerebellar neurons and their abnormal intracortical circuits. This study is basically important to understand the pathogenesis of clinical cerebellar syndromes, such as gait disturbance, tremor, nystagmus, compression of cranial nerves, cognitive and neurobehavioural changes observed in the affected patients.

\section{Material and methods}

The present study describes the ultrastructural changes of focal peritumoural cerebellar cortex by means of cortical biopsies immediately taken during neurosurgical treatment of seven patients with cerebellar, cerebellopontine and mesencephalic tumours. Table I contains the clinical data, neuropathological features and localization of tumours. The neurosurgical study was performed and the cortical biopsies were taken according to basic principles of Helsinki. Cerebellar cortical biopsies of seven patients, ranging from 2- to 50-years old, with clinical diagnosis of cerebellar haemangioma, mesencephalic meningioma, cerebellopontine astrocytoma, cerebellopontine meningioma, and medulloblastoma of cerebellar vermis were examined by means of conventional transmission electron microscopy. Two to five $\mathrm{mm}$ thick cortical biopsies were immediately fixed in the surgical room in $4 \%$ glutaraldehyde- $0.1 \mathrm{M}$ phosphate or cacodylate buffer, $\mathrm{pH} 7.4$, at $4^{\circ} \mathrm{C}$. After 2 hours' glutaraldehyde-fixation period, the cortical biopsies were divided into approximately $1 \mathrm{~mm}$ fragments and observed under a stereoscopic microscope to check the quality of fixation of the sample, the glutaraldehyde diffusion rate, and the brownish coloration of the surface and deeper cortical regions, indicative of good glutaraldehyde fixation by immersion technique. Immersion in fresh glutaraldehyde solution of $1 \mathrm{~mm}$ slices was secondarily done for 2 hours after eliminating the remaining blood from the cortical biopsy by washing in similar $0.1 \mathrm{M}$ phosphate or cacodylate buffer, $\mathrm{pH} 7.4$ to avoid oxidation of the primary fixative solution. Secondary fixation in $1 \%$ osmium tetroxide- $0.1 \mathrm{M}$ phosphate buffer, pH 7.4, was carried out for 1-2 hours at $4^{\circ} \mathrm{C}$. Black staining of the cortical slices also was observed under a stereoscopic microscope to check osmium tetroxide diffusion rate and quality of secondary fixation. They were then rinsed for 5 to 10 minutes in phosphate or cacodylate buffer of similar composition to that used in the fixative solution, dehydrated in increasing concentrations of ethanol, and embedded in Araldite or Epon. For proper orientation during the electron microscope study and observation of cortical layers, approximately 0.1 to $1 \mu \mathrm{m}$ thick sections were stained with toluidine blue and examined with a Zeiss photomicroscope. Ultrathin sections, obtained with Porter-Blum and LKB ultramicrotomes, were stained with uranyl acetate and lead citrate, and observed in a JEOL $100 \mathrm{~B}$ transmission electron microscope (TEM) at magnifications ranging from $20,000 \times$ to $90,000 \times$. For each cortical biopsy, approximately 50 electron micrographs were systematically studied. Digital images were Photoshop analysed [7]. This paper was carried out according to the ethical principles of Helsinki Declaration, Ethical Committee of Biological Research Institute. The relative written consent was obtained in each case.

Table I. Neurosurgical study of oedematous human cerebellum

\begin{tabular}{|lll|}
\hline Case No. 1: MRR, 45 y, M & $\begin{array}{l}\text { Tremor in upper and lower limbs, incoherent } \\
\text { speech, gait disturbance, visual hallucinations, } \\
\text { clouded sensorium, stupor }\end{array}$ & Medulloblastoma of cerebellar vermis \\
\hline Case No. 2: ARM, 30 y, M & Headache, dismetry, gait disturbance and tremor & Cerebellopontine angle astrocytoma \\
\hline Case No. 3: MIJ, 50 y, F & Headache, dismetry, tremor and ataxic gait & Cerebellar haemangioma cavernosum \\
\hline Case No. 4: EJPV, 10 y, M & Dismetry, tremor, ataxic gait & Cerebellopontine angle meningioma \\
\hline Case No. 5: GPRM, 2 y, F & $\begin{array}{l}\text { Headache, vomits, nystagmus, } \\
\text { paralysis of VI cranial nerve and ataxic gait }\end{array}$ & Medulloblastoma of cerebellar vermis \\
\hline Case No. 6: FMQ, 9 y, F & Headache, tremor, nystagmus and ataxic gait & Cerebellopontine angle meningioma \\
\hline Case No. 7: AML, 25 y, M & Headache, nystagmus, tremor and ataxia & Mesencephalic meningioma \\
\hline
\end{tabular}




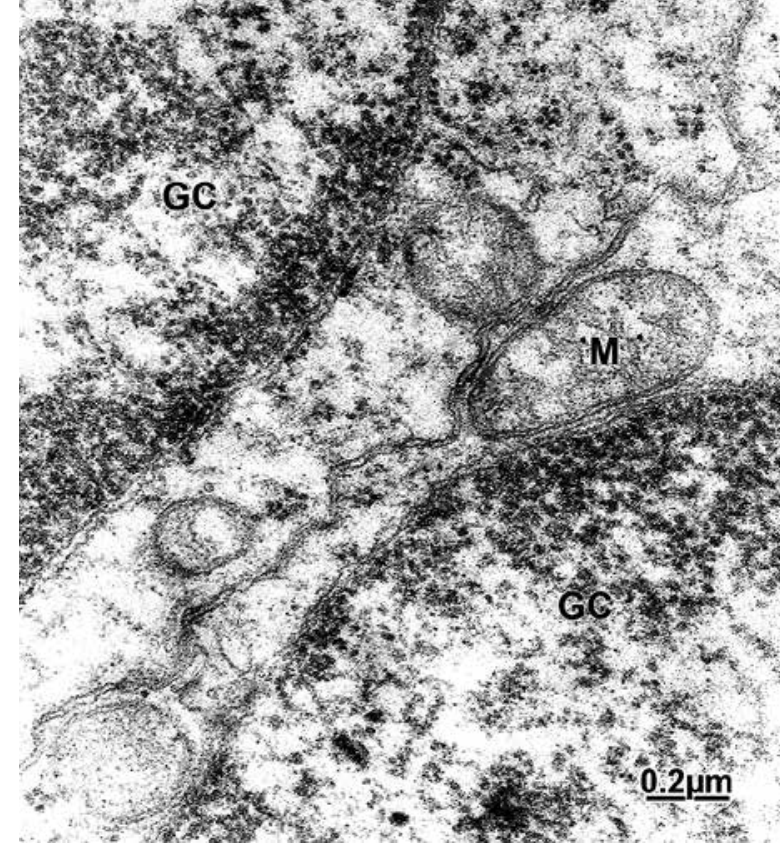

Fig. 1. Cerebellar medulloblastoma. Cerebellar cortex granular layer. Two swollen granule cells (GC) showing oedematous mitochondria (M). Note the twisted course of confronting limiting plasma membranes due to the expansive forces induced by the tumour.

\section{Results}

Close examination of cerebellar cortex showed at the level of granule cell layer, swollen granule cells characterized by an electron translucent cytoplasm and swollen mitochondria. There appeared closely apposed and their continuous limiting plasma membrane appeared separated by a $20 \mathrm{~nm}$ extracellular space (Fig. 1). The neighbouring mossy fibre glomerulus showed the oedematous afferent mossy fibre endings synapsing with swollen granule cell dendrites. Degenerated myelinated axons were observed at the granular layer. Some of these degenerated axons could correspond to the efferent Purkinje cell axons and the incoming afferent cerebellar mossy and climbing fibres (Fig. 2). Reactive astrocytes containing an increased amount of huge lipofuscin granules were found at the granular and molecular layers (Fig. 3).

The oedematous Golgi cell depicted an electron translucent cytoplasm, notably swollen mitochondria, dilated rough endoplasmic reticulum cisterns with focal detachment of associated ribosomes, enlarged

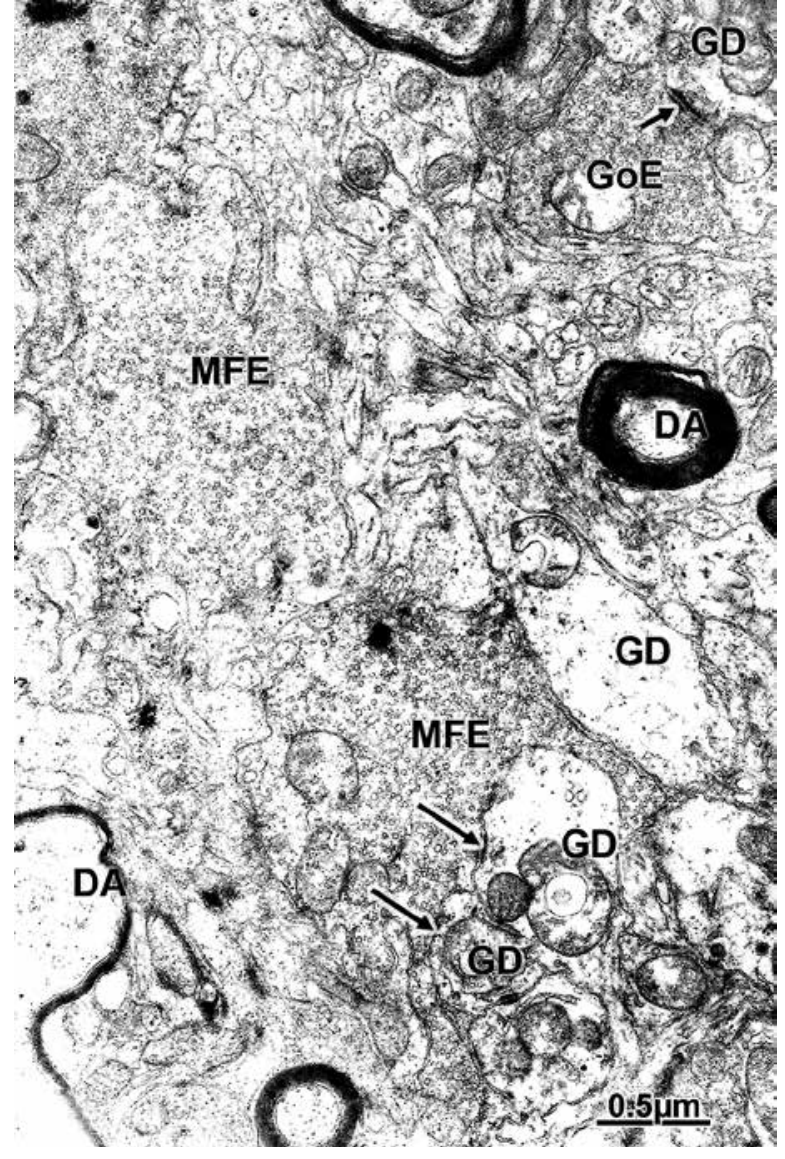

Fig. 2. Cerebellar meningioma. Cerebellar cortex granular layer. Notably swollen mossy fibre ending (MFE) containing a central core of oedematous mitochondria and numerous clear spheroidal synaptic vesicles. The long arrows indicate the synaptic contacts with granule cell dendritic tips (GD). A Golgi cell synaptic ending (GoE) localized at the periphery of cerebellar glomerulus appears making asymmetric synaptic contact (short arrow) with a granule dendrite (GD). Neighbouring degenerated myelinated axons (DA) corresponding to cerebellar afferent and/or efferent fibres also are distinguished.

flattened cisterns of Golgi apparatus, numerous lipofuscin granules, and intranuclear inclusions (Fig. 4).

At the level of Purkinje cell layer, clear and dark ischaemic Purkinje cell bodies exhibited a dilated smooth and rough endoplasmic reticulum with focal detachment of membrane associated ribosomes (Figs. 5 and 6). The Purkinje cell bodies appeared covered by the swollen Bergmann glial cell cytoplasm. Dense Purkinje cell dendrites can be traced 


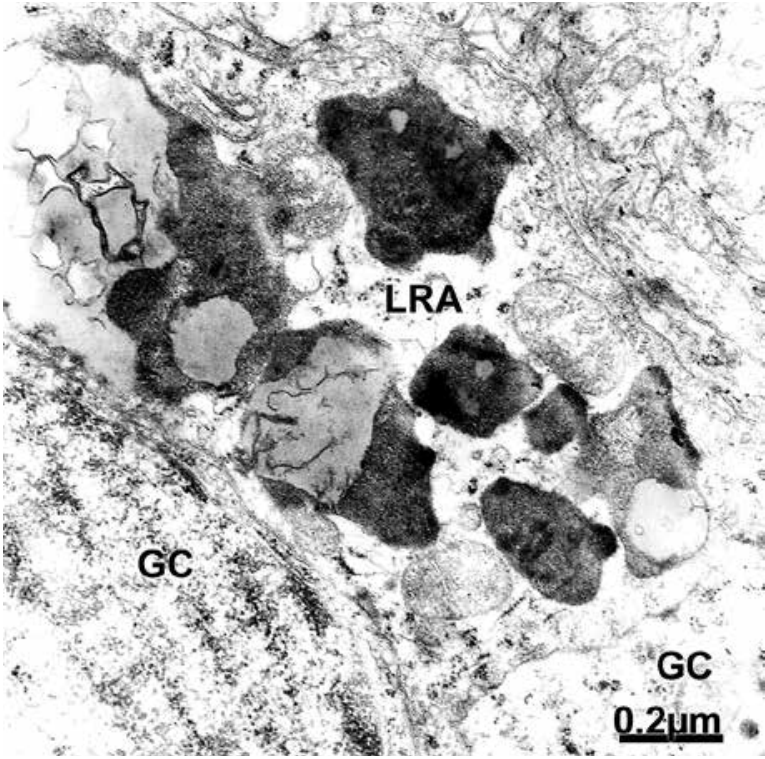

Fig. 3. Cerebellar meningioma. Cerebellar cortex granular layer. Lipofuscin-rich astrocyte (LRA) showing numerous and huge lipofuscin granules. The neighbouring swollen granule cells (GC) also are seen.

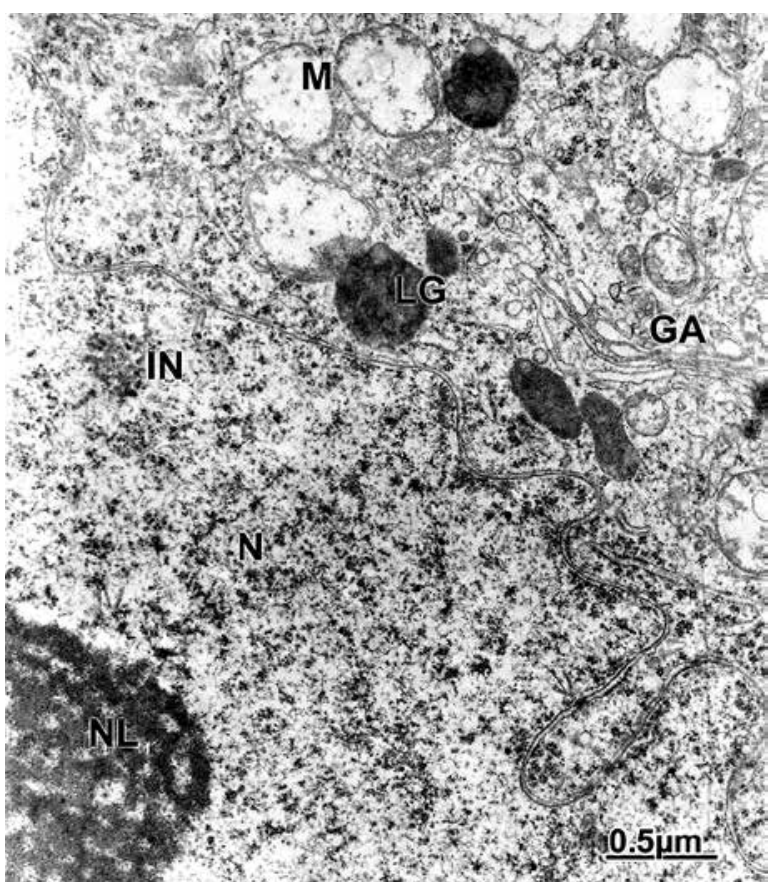

Fig. 4. Cerebellar haemangioma. Cerebellar cortex granular layer. Oedematous and clear Golgi cell soma (GC) exhibiting a lobulated nucleus and prominent nucleolus (NL), dilated rough endoplasmic reticulum (ER), and Golgi apparatus (GA), clustered free ribosomes and polyribosomes, lipofuscin granules (LG), and notably swollen mitochondria $(M)$.

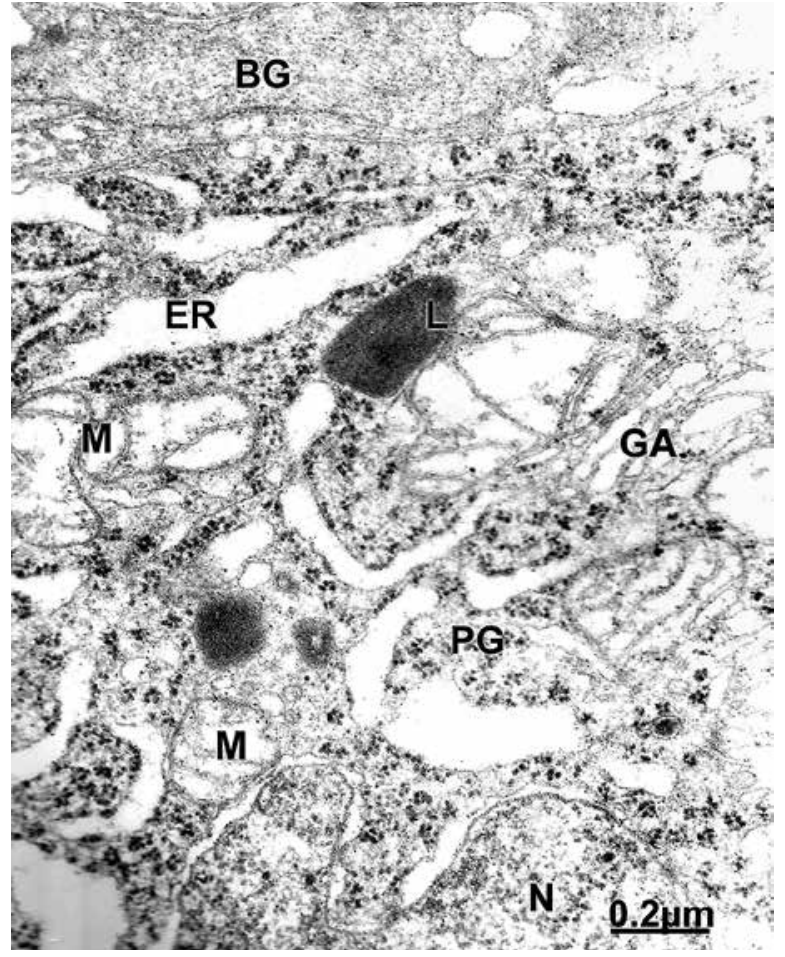

Fig. 5. Cerebellar meningioma. Cerebellar cortex Purkinje cell layer. Ischaemic and clear Purkinje cell body exhibiting a lobulated nucleus $(\mathrm{N})$, and the irregularly dilated nuclear envelope, swollen rough (ER) endoplasmic reticulum with detachment of associated ribosomes, dense lysosomes $(L)$, and swollen both mitochondria $(M)$, and Golgi apparatus (GA), swollen mitochondria (M) and Golgi apparatus (GA), and lysosomes (L) with dark and fine granular matrix deposits. The satellite oedematous Bergmann glial cell $(B G)$ is seen covering the Purkinje cell body.

throughout the width of molecular layer exhibiting the emergency sites of Purkinje dendritic spines, which establish axo-spino-dendritic synaptic contacts with the swollen synaptic varicosities of parallel fibres (Fig. 7). Oedematous Bergmann glial cells were observed containing swollen mitochondria and lipofuscin granules, and exhibiting the characteristic ascending Bergman fibres in the molecular layer (Fig. 8). The cytoplasm of Bergman glial cell also appeared surrounding the Purkinje recurrent axonal collaterals. In addition, swollen basket cells were found surrounding the Purkinje cell layer (Fig. 9).

The oedematous stellate neurons displayed swollen mitochondria, invaginated nuclear envelope, and 


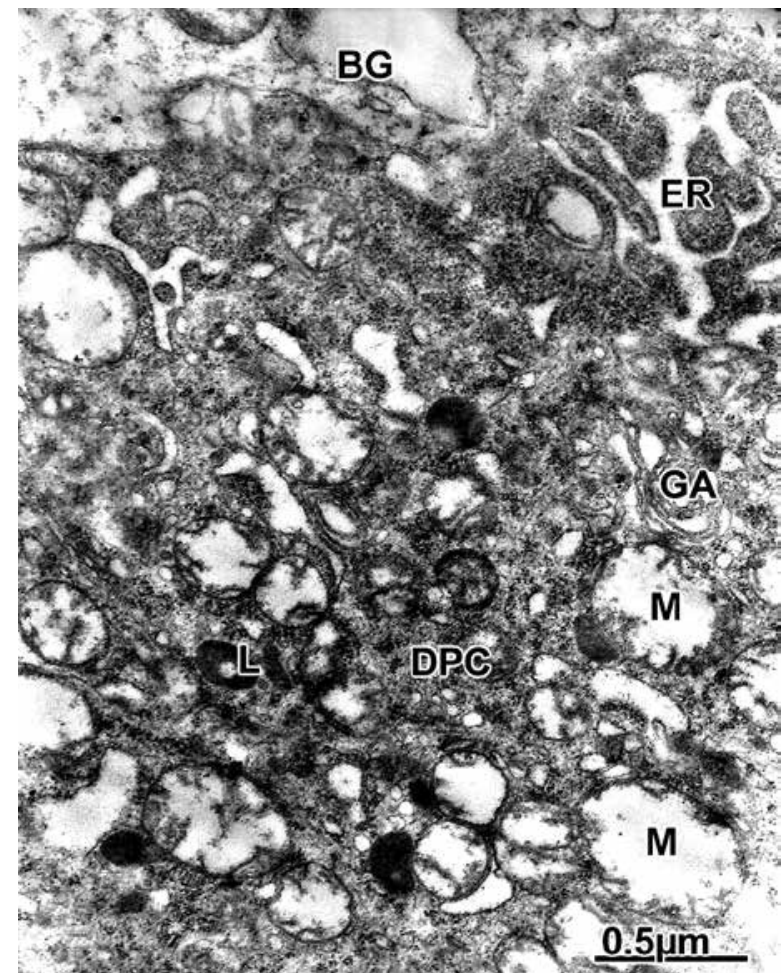

Fig. 6. Cerebellar meningioma. Dark Purkinje cell (DPC) body showing the dense cytoplasmic matrix, tortuous aspect of rough endoplasmic reticulum (ER), the notably swollen mitochondria with fragmented cristae $(M)$, oedematous Golgi apparatus (GA), vacuolated lysosomes (L). The oedematous and vacuolated Bergmann glial cell (BG) also is seen.

enlarged rough endoplasmic reticulum with detachment of associated ribosomes (Fig. 10). Proteinaceous oedema fluid with fibrinous organization was found occupying the enlarged extracellular space of cerebellar molecular layer (Fig. 11).

Swollen longitudinal granule cell axons or parallel and climbing fibres were observed at the molecular layer alternating with the ascending and swollen Bergmann glial fibres, and clear dendritic processes of Golgi, basket and stellate cells. The parallel fibres were identified by the characteristic cross-sectioned bundles of parallel fibres and their degenerated "en passant" synaptic varicosities. In addition, the swollen cytoplasm of Bergman glial cell closely surrounds the degenerated parallel fibre-Purkinje dendritic spine synapses. The latter appeared as dark degenerated structures (Fig. 12). Swollen climbing fibre branches appeared as electron translucent unmy-

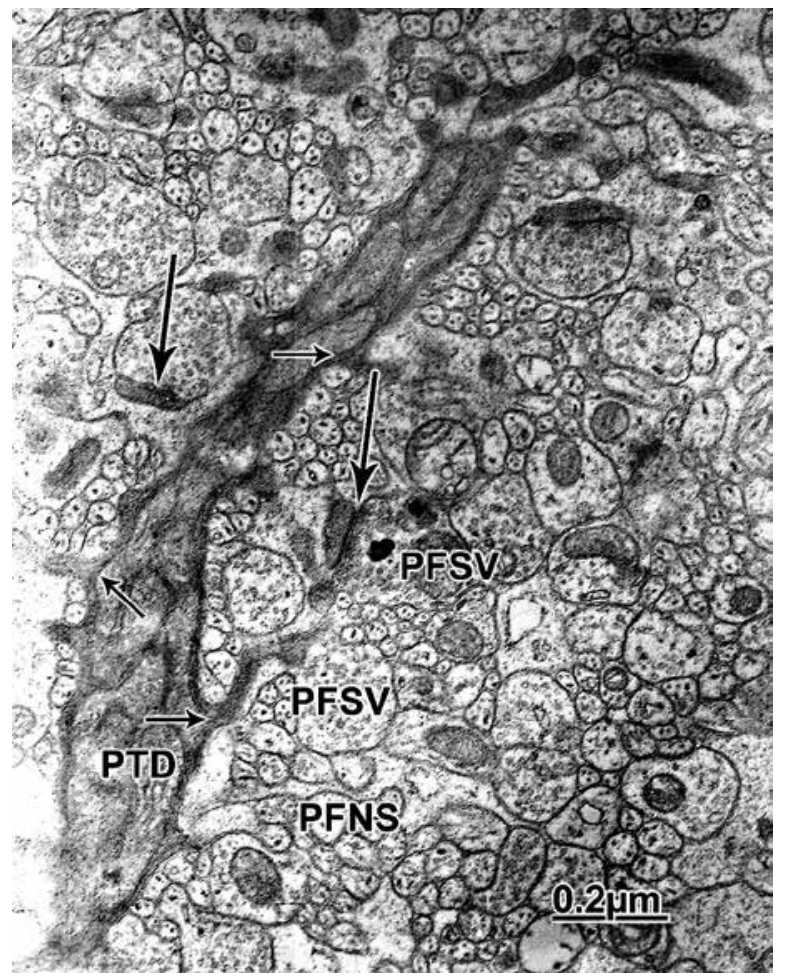

Fig. 7. Cerebellar meningioma. Cerebellar molecular layer outer surface showing a dark tertiary Purkinje dendrite (PTD) showing the sites of emergency of dark dendritic spines (short arrows), and the degenerated axo-spinodendritic contacts of parallel fibre varicosities with Purkinje dark dendritic spines (long arrows). The cross sections of non-synaptic segments (PFNS) of parallel fibres also are distinguished.

elinated axons ascending in the molecular layer and containing numerous microtubules and neurofilaments. The large climbing fibre synaptic ending showed a decreased number of synaptic vesicles closely aggregated toward the presynaptic membrane. They appeared making synaptic contacts with clear and oedematous processes, presumably corresponding to ascending dendrites of Golgi, stellate and basket cell dendrites. Swollen and reactive Bergmann glial cells were observed surrounding climbing fibre endings (Fig. 13).

Swollen and dark microglial cells were observed engulfing haematogenous serum proteinaceous oedema fluid (Fig. 14). Some cerebellar capillaries showed a reduplicated basement membrane (Fig. 15), and open and apparently intact endothelial junctions. Similar findings also were reported by us in traumatic brain injuries [7]. 


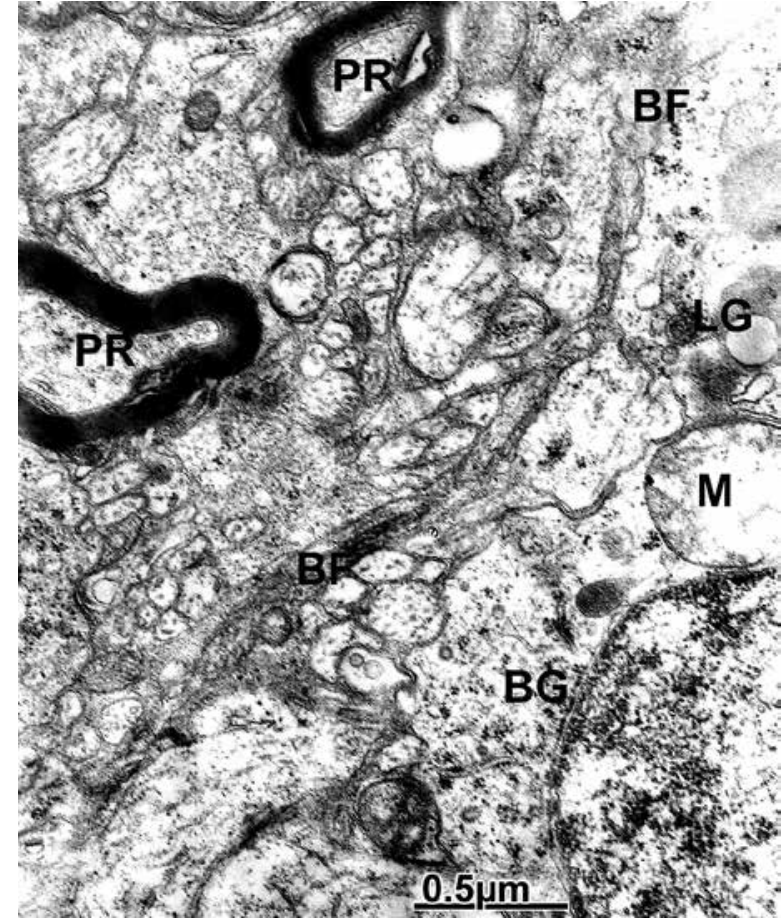

Fig. 8. Cerebellar medulloblastoma. Cerebellar Purkinje cell layer. Swollen Bergmann glial cell (BGC) showing oedematous mitochondria (M) and lipofuscin granules (LG). The ascending Bergmann fibres (BF) and Purkinje recurrent axonal collaterals (PR) also are seen.

\section{Discussion}

The present study describes the oedematous intracellular changes and degenerated intracortical circuits of cerebellar neurons, and the reactive changes and neuroglial cells of cerebellar cortex in different cerebellar and brain stem tumours examined. Swollen clear granule, Golgi, Purkinje, stellate and basket cells, and Bergmann glial were found. On the contrary, some dark ischaemic Purkinje cells were observed suggesting a major vulnerability of Purkinje cells to the tumour expansion forces and the sustained ischaemic process. Similar results in cerebellar neurons, mainly dark Purkinje cells and Bergmann glial cells as described above, were also reported earlier by Sobaniec-Lotowska [28] in experimental encephalopathy induced by chronic application of valproate. According to this author, the general pattern of submicroscopic alterations of Purkinje cell perikarya suggested severe disorders in several intercellular biochemical extents, including

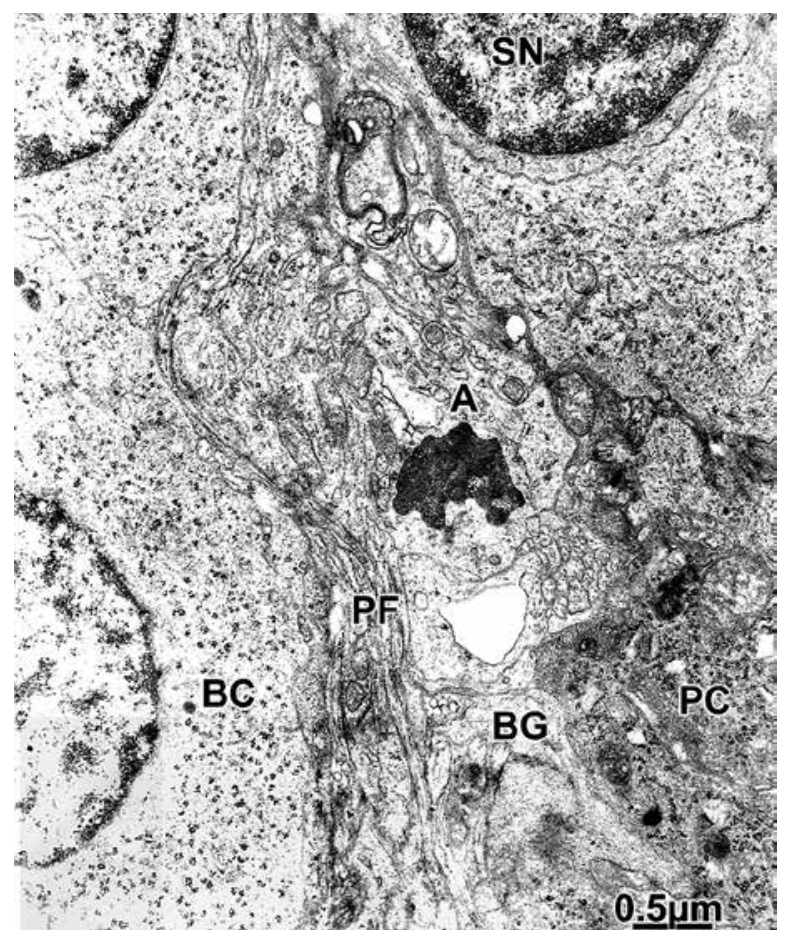

Fig. 9. Cerebellar meningioma. Cerebellar cortex Purkinje cell layer. Swollen basket cells (BC) surrounded by segments of swollen Bergmann glial cell (BG), dark Purkinje cell (PC) and lipofuscin-rich astrocyte (A) cytoplasm. The profile of parallel fibre bundles (PF) ascending toward the molecular layer, and the stellate neurons (SN) also are distinguished.

inhibition of oxidative phosphorylation and abnormal protein synthesis, both of which could lead to lethal damage. The enlargement of smooth and endoplasmic reticulum (ER) and the focal detachment of associated ribosomes suggest a form of stress-induced endoplasmic reticulum dysfunction [16]. According to Paschen [26], changes in neuronal calcium activity in the various subcellular compartments have divergent effects on affected cells. In the cytoplasm and mitochondria, where calcium activity is normally low, a prolonged excessive rise in free calcium levels is believed to be toxic. On the contrary, in the endoplasmic reticulum, calcium activity is relatively high and severe stress is caused by a depletion of ER calcium stores.

Dark Purkinje cell dendritic branches and dendritic spines were observed at the molecular layer. These findings herein interpreted as characterizing ischaemic Purkinje neurons, could also be due to acti- 


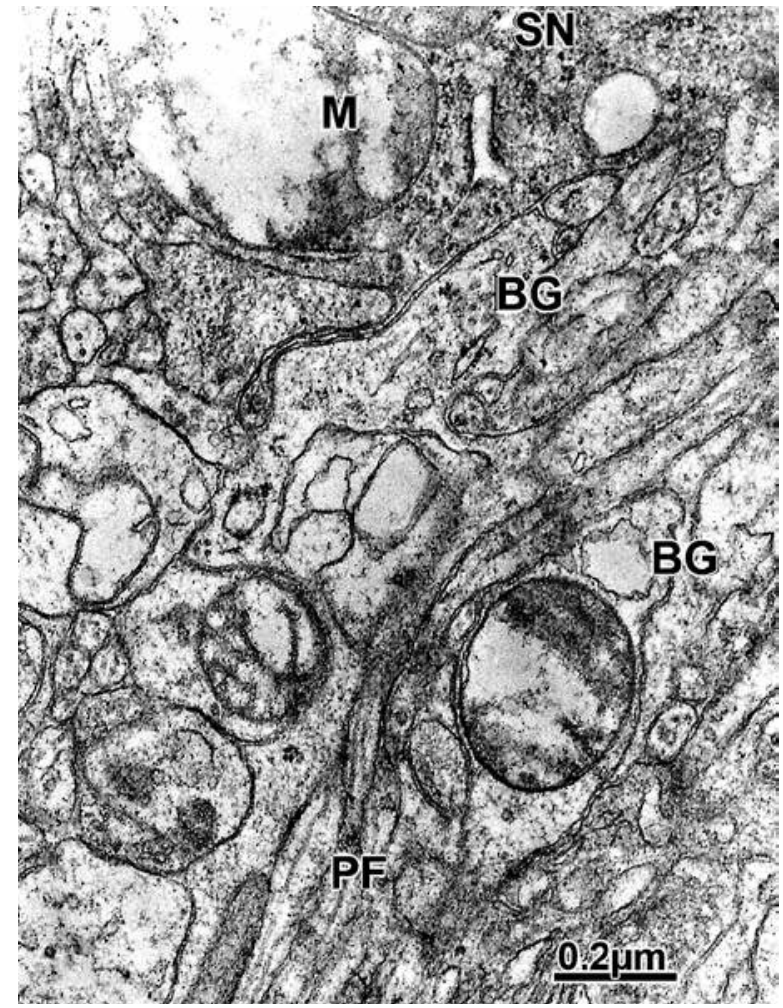

Fig. 10. Cerebellar hemangioma. Cerebellar molecular layer. Notably swollen stellate neuron (SN) with a degenerated mitochondrion (M), and surrounded by swollen and vacuolated Bergmann glial cell cytoplasm (BG) and ascending parallel fibre bundles (PF).

vation of caspases and mitochondrial dysfunction [31], or hypoxia induced excitotoxic-type of dark cell degeneration [2]. Dark Purkinje dendritic arborisation and the degenerated parallel fibre and climbing Purkinje spines dendritic synapses could be related to the cognitive impairment and motor deficits of patients under study. Similar findings have been reported on dendritic Purkinje cells from human cerebellar vermis in Alzheimer's disease [25].

The presence of intranuclear filamentous inclusions in cerebellar Golgi cells was earlier reported by Castejon and Arismendi [4] in three patients with cerebellar tumours. They were related with stress conditions, excitotoxicity, damage of mitochondrial respiratory chain and impaired energy metabolism [18].

Degenerated cerebellar afferent and efferent myelinated axons were found in the granular and molecular layers of cerebellar cortex, which provide

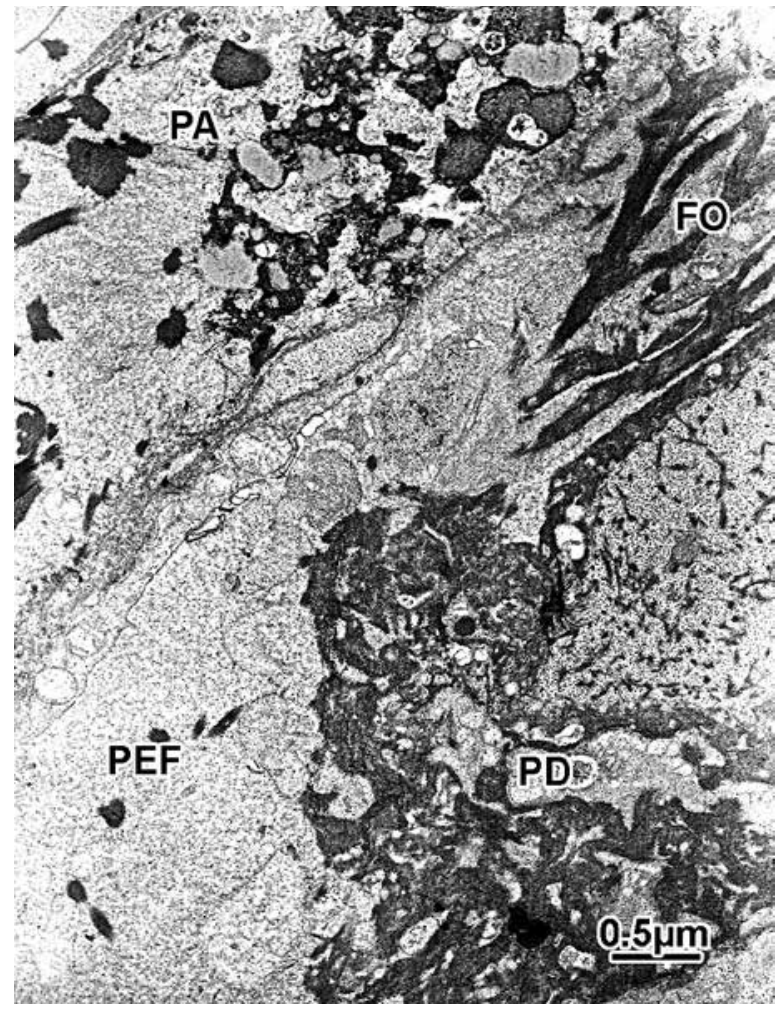

Fig. 11. Cerebellar cavernous haemangioma. Proteinaceous oedema fluid (PEF) deposited in the enlarged extracellular space exhibiting fibrinous organization (FO) and surrounded by a dark Purkinje cell degenerated dendrite (PD) and a phagocytic and lipofuscin-rich astrocyte (PA) containing deposits of fibrinous material.

evidence for myelin lipid dysfunction in the oedematous cerebellar cortex related with the oligodendrocyte degenerating hydropic changes [6,13]. Cytoskeletal disassembly and caspases are recently implicated in these processes highlighting the degenerating axonal processes in human hypoxic- ischaemic injury [29].

The presynaptic synaptic terminals of climbing fibres synapsing on Purkinje cell dendrites exhibited a decreased number of synaptic vesicles, indicating climbing fibre presynaptic degeneration [11]. Similar findings were reported by Baloyannis in vascular dementia [1].

We found lipofuscin-rich astrocyte which can be related with the associated ischaemic and degenerative processes. Similar observations have been reported by the present author in traumatic human brain injuries $[9,10]$. We also found reactive phagocytic astrocytes and microglial cells engulfing hae- 


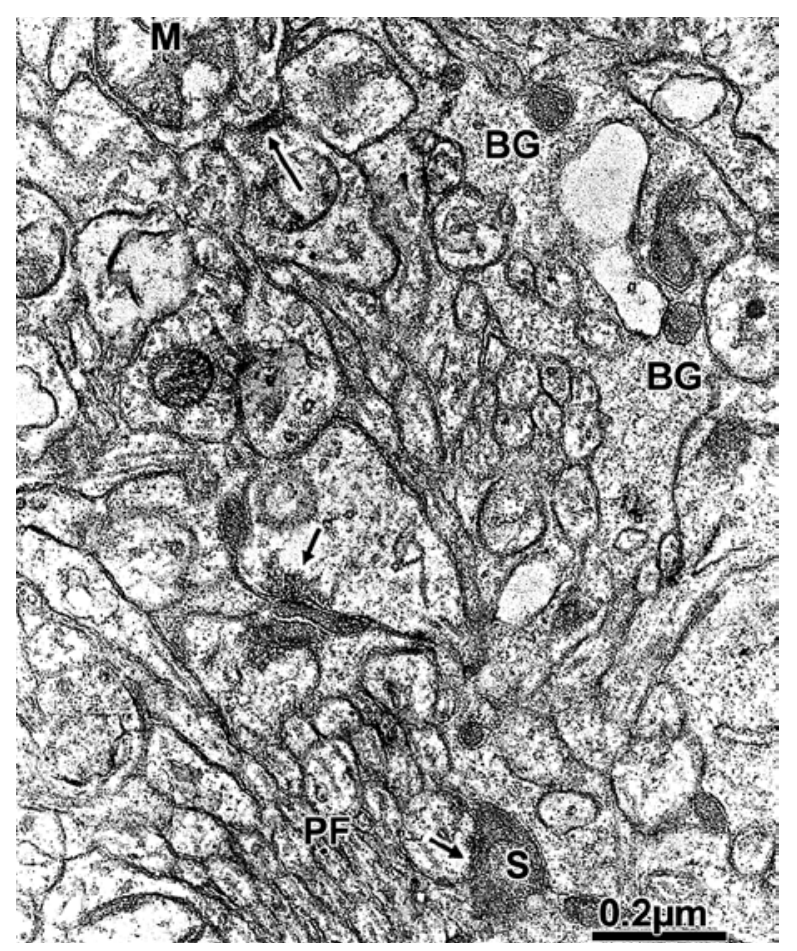

Fig. 12. Cerebellar meningioma. Cerebellar molecular layer showing the degenerated parallel fibredark Purkinje cell dendritic spine synapses (short arrows) and the degenerated climbing fibre-Purkinje dendrite synapses (long arrows). Proteinrich Bergmann glial cell (BG) swollen cytoplasm surrounds the molecular layer neuropil.

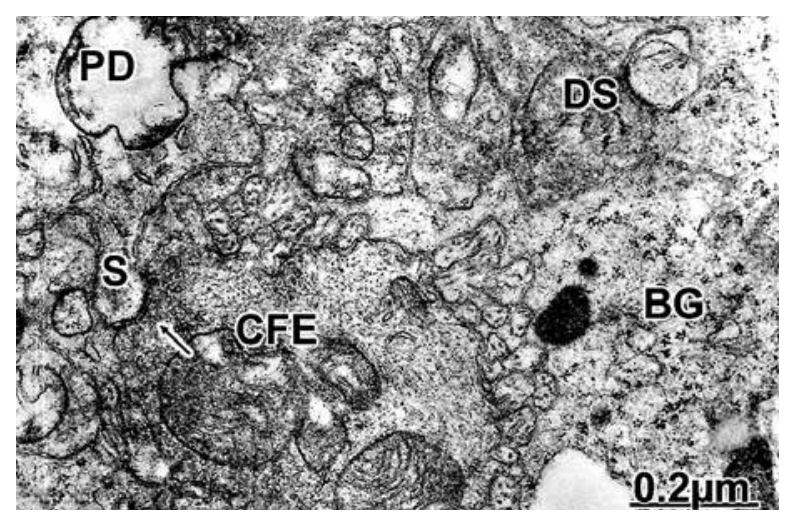

Fig. 13. Cerebellar hemangioma. Large and swollen climbing fibre ending (CFE) containing neurofilaments, swollen clear mitochondria and scarce synaptic vesicles making a swollen axo-spinodendritic contact (arrow) with a degenerated spine (S) of a swollen and clear Purkinje cell dendrite (PD). Note the enveloping perisynaptic Bergmann glial cell cytoplasm (BG). The upper right side of the figure shows degenerated presynaptic endings (DS).

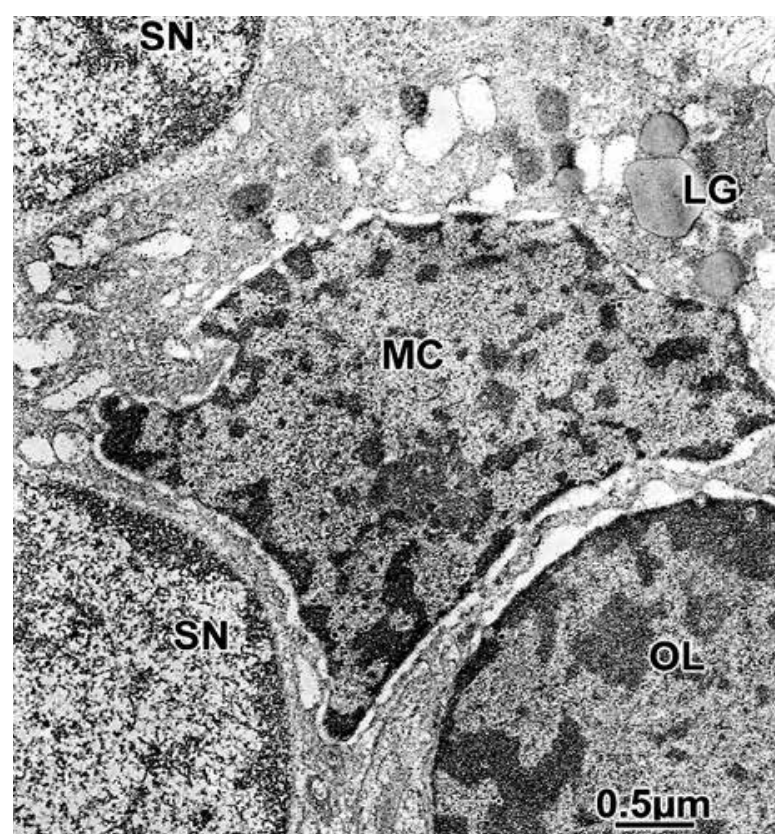

Fig. 14. Cerebellar meningioma. Cerebellar molecular layer showing a swollen and phagocytic microglial cell (MC) containing lipofuscin granules (LG) and phagocytic vacuoles, and a swollen oligodendrocyte (OL) intercalated among stellate neurons (SN).

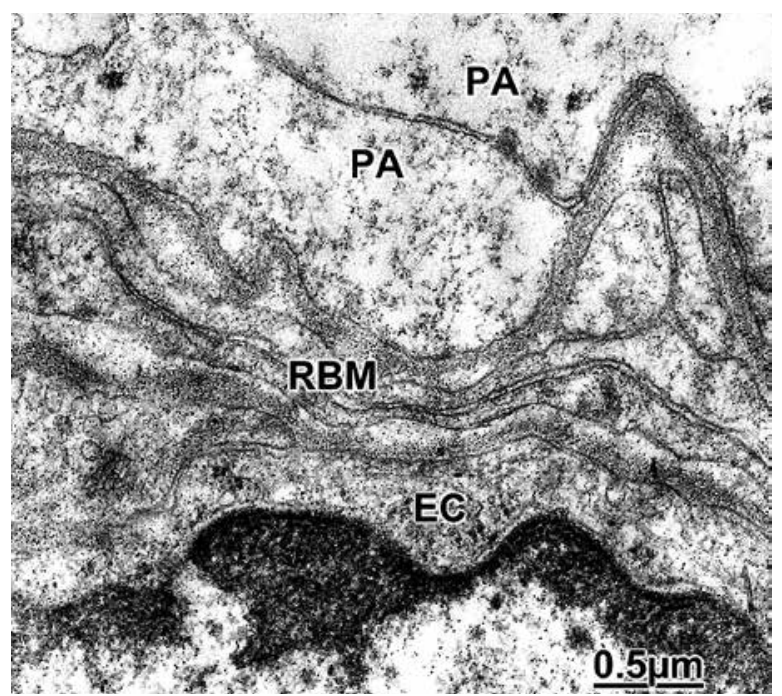

Fig. 15. Cerebellar meningioma. Cerebellar molecular layer. Cerebellar capillary showing the swollen abluminal cytoplasm of endothelial cell (EC), the reduplicated, swollen and vacuolated basement membrane (BM) exhibiting fine granular deposits of degraded glycoproteins basement matrix, and the oedematous perivascular astrocytic end-feet (PA). The swollen pericyte cytoplasm is observed between the basement membrane layering. 
matogenous proteinaceous oedema fluid. These findings have been also described by Stoll et al. [30] in ischaemic brain lesions, and by us in severe traumatic and complicated human brain injuries $[5,9,10]$.

\section{Clinical correlates of peritumoural ischaemic process}

The electron microscopic findings described herein and related with the oedematous microneurons (granule, basket, and stellate cells) and macroneurons (Golgi and Purkinje cells) of cerebella cortex, and their degenerated synaptic contacts in both granule cell and molecular layers with cerebellar afferent and intrinsic fibres could be related with the tremor, nystagmus, dismetry, gait disturbance and neurobehavioral changes observed in the patients examined. According to Louise [21], a cascade of biochemical and cellular events occurring in the Purkinje cell layer and its neuron neighbourhood, as well as the physiological effects of secondary remodelling/rewiring could be related with essential tremor. The derangement of neurons and intracortical circuits is in agreement with the hypothesis that cerebellar damage impairs executive control and monitoring of movement generation [3].

\section{Some molecular considerations on peritumoural cerebellar ischaemic process}

The peritumoural ischaemic process seems to include the detrimental biochemical events postulated by Hou and MacManus [17] for ischaemia-induced neuronal death, such as perturbation of calcium homeostasis leading to increased excitotoxicity, malfunction of endoplasmic reticulum and mitochondria, elevation of oxidative stress causing DNA damage, alteration in proapoptotic gene expression, and activation of the effector cysteine proteases (caspases) and endonucleases leading to the final degradation of the genome. The observed peritumoural cerebellar cell changes herein described also are similar to those described as permanent compression ischaemia [19]. A role of glutamate receptors in ischaemic process has been firmly established and a prominent characteristic of ischaemic insults is endoplasmic reticulum (ER) stress. In addition, a down-regulation of cell surface GABAB receptors has been postulated leading to diminished neuronal inhibition and contributing to excitotoxicity in cerebral ischaemia [23]. Cerebellar Purkinje neurons are selectively vulnerable to AMPA (alpha-amino-3hydroxy-5-methyl-4-isoxazolepriopionic acid)-induced delayed neurotoxicity known as dark cell degeneration that is expressed as cytoplasmic and nuclear condensation, neuron shrinkage, and failure of physiology [31]. Sustained activation of N-methyl-d-aspartate (NMDA)-type glutamate receptors leads to excitotoxic neuronal death as observed in stroke, brain trauma, and neurodegenerative disorders. Superoxide production by NADPH oxidase is a requisite event in the process leading from NMDA receptor activation to excitotoxic death [20].

Alterations of cerebellar excitatory and inhibitory synaptic transmission may contribute to the ischaemia-induced neuronal degeneration [22]. We have observed swollen mitochondria indicating a decrease in high energy phosphate produced by ischaemia, which causes depletion of the materials necessary to produce this phosphate and strongly affecting the electron transport chain. The biochemical pathology of swollen mitochondria in brain oedema have been earlier analysed in detail by us in a previous publication [12].

The presence of extracellular electron dense deposits of proteinaceous oedema fluid and fibrinous organization, and the reduplicated capillary basement layering have also been described by us in severe traumatic brain injuries $[7,8]$.

\section{Acknowledgments}

This paper has been carried out with a subvention obtained from CONDES-LUZ and Castejón Foundation. We deeply appreciate the technical digital help of Lic. Orlando Castejon de Pablos for preparation of electron micrographs.

\section{Disclosure}

Author reports no conflict of interest.

\section{References}

1. Baloyannis SJ. Pathological alterations of the climbing fibres of the cerebellum in vascular dementia: a Golgi and electronmicroscope study. J Neurol Sci 2007; 257: 56-61.

2. Barenberg P, Strahlendorf $\mathrm{H}$, Strahlendorf J. Hypoxia induces an excitotoxic-type of dark cell degeneration in cerebellar Purkinje neurons. Neurosci Res 2001; 40: 245-254.

3. Brunamonti E, Chiricozzi FR, Clausi S, Olivito G, Giusti MA, Molinari $M$, Ferraina $S$, Leggio $M$. Cerebellar damage impairs exec- 
utive control and monitoring of movement generation. PLoS One 2014; 9: e85997.

4. Castejón OJ, Arismendi GJ. Intranuclear filamentous inclusion in human oedematous cerebellar Golgi cells. J Submicrosc Cytol Pathol 2003; 35: 389-393.

5. Castejón OJ. Electron microscopy of astrocyte changes and subtypes in traumatic human edematous cerebral cortex: a review. Ultrastruct Pathol 2013; 37: 417-424.

6. Castejón OJ. Electron microscopic study of central axona degeneration in traumatic human brain edema. J Submicrosc Cytol 1985; 17: 703-718,

7. Castejón OJ, Valero C, Díaz M. Light and electron microscopy of nerve cells in traumatic oedematous human cerebral cortex. Brain Inj 1997; 11: 363-388.

8. Castejón OJ. Ultrastructural alterations of human cortical cap illary basement membrane in perifocal brain edema. A review. Folia Neuropathol 2014; 52: 10-21.

9. Castejón OJ. Morphological astrocytic changes in complicated human brain trauma. A light and electron microscopic study. Brain Inj 1998; 12: 409-427.

10. Castejón OJ. Astrocyte subtypes in the gray matter of injured human cerebral cortex. A transmission electron microscopic study. Brain Inj 1999; 13: 291-304.

11. Castejón OJ, Valero C, Díaz M. Synaptic degenerative changes in human traumatic brain edema. J Neurosurg Sci 1995; 39: 47-65.

12. Castejón OJ. Structural pattern of injured mitochondria in oedematous human cerebral cortex. Brain Inj 2004; 18: 1107-1126.

13. Castejón OJ. Ultrastructural pathology of oligodendroglial cells in traumatic and hydrocephalic human brain edema: a review. Ultrastruct Pathol 2015; 39: 359-368.

14. Chaudhry AP, Montes M, Cohn GA. Ultrastructure of cerebellar hemangioblastoma. Cancer 1978; 42: 1834-1850.

15. Ferrer I, Isamat F, Acebes J. A Golgi and electron microscopic study of a dysplastic gangliocytoma of the cerebellum. Acta Neuropathol 1979; 47: 163-165.

16. Ho KL. Ultrastructure of cerebellar capillary hemangioblastoma. IV. Pericytes and their relationship to endothelial cells. Acta Neuropathol 1985; 67: 2542-2564.

17. Hou ST, MacManus JP. Molecular mechanisms of cerebral ischemia-induced neuronal death. Int Rev Cytol 2002; 221: 93-148.

18. lijima T. Mitochondrial membrane potential and ischemic neuronal death. Neurosci Res 2006; 55: 234-243.

19. Kalimo H, Paljärvi L, Vapalahti M. The early ultrastructural alterations in the rabbit cerebral and cerebellar cortex after compression ischaemia. Neuropathol Appl Neurobiol 1979; 5: 211-223.

20. Lam TI, Brennan-Minnella AM, Won SJ, Shen Y, Hefner C, Shi Y, Sun $\mathrm{D}$, Swanson RA. Intracellular $\mathrm{pH}$ reduction prevents excitotoxic and ischemic neuronal death by inhibiting NADPH oxidase. Proc Natl Acad Sci U S A 2013; 110: 362-368.

21. Louis ED. From neurons to neuron neighborhoods: the rewiring of the cerebellar cortex in essential tremor. Cerebellum 2014, 13: 501-512.

22. Liang R, Pang ZP, Deng P, Xu ZC. Transient enhancement of inhibitory synaptic transmission in hippocampal CA1 pyramidal neurons after cerebral ischemia. Neuroscience 2009; 160: 412-418.

23. Maier PJ, Zemoura K, Acuña MA, Yévenes GE, Zeilhofer HU, Benke D. Ischemia-like oxygen and glucose deprivation mediates down-regulation of cell surface $\gamma$-aminobutyric acidB receptors via the endoplasmic reticulum (ER) stress-induced transcription factor CCAAT/enhancer-binding protein (C/EBP)-homologous protein (CHOP). J Biol Chem 2014; 289: 12896-12907.

24. Mann DM, Stamp JE, Yates PO, Bannister CM. The fine structure of the axonal torpedo in Purkinje cells of the human cerebellum. Neurol Res 1980; 1: 369-378.

25. Mavroudis IA, Manani MG, Petrides F, Petsoglou K, Njau SD, Costa VG, Baloyannis SJ. Dendritic and spinal pathology of the Purkinje cells from the human cerebellar vermis in Alzheimer's disease. Psychiatr Danub 2013; 25: 221-226.

26. Paschen W. Endoplasmic reticulum: a primary target in various acute disorders and degenerative diseases of the brain. Cell Calcium 2003; 34: 365-383.

27. Probst A, Ulrich J, Zdrojewski B, Hirt HR. Cerebellar ganglioglioma in a child. J Neuropathol Exp Neurol 1979; 38: 57-71.

28. Sobaniec-Lotowska ME. Ultrastructure of Purkinje cell perikarya and their dendritic processes in the rat cerebellar cortex in experimental encephalopathy induced by chronic application of valproate. Int J Exp Pathol 2001; 82: 337-348.

29. Sokolowski JD, Gamage KK, Heffron DS, Leblanc AC, Deppmann CD, Mandell JW. Caspase-mediated cleavage of actin and tubulin is a common feature and sensitive marker of axonal degeneration in neural development and injury. Acta Neuropathol Commun 2014; 2: 16.

30. Stoll G, Jander S, Schroeter M. Inflammation and glial responses in ischemic brain lesions. Prog Neurobiol 1998; 56: 149-171.

31. Strahlendorf J, Box C, Attridge J, Diertien J, Finckbone V, Henne WM, Medina MS, Miles R, Oomman S, Schneider M, Singh H, Veliyaparambil M, Strahlendorf $H$. AMPA-induced dark cell degeneration of cerebellar Purkinje neurons involves activation of caspases and apparent mitochondrial dysfunction. Brain Res 2003; 994: 146-159.

32. Yagishita S. Morphological investigations on axonal swellings and spheroids in various human diseases. Virchows Arch A Pathol Anat Histol 1978; 378: 181-197. 